\title{
Analysis of the heteroplasmy level and transmitted features in hearing-loss pedigrees with mitochondrial 12S rRNA A1555G mutation
}

Yuhua Zhu', Shasha Huang ${ }^{1}$, Dongyang Kang ${ }^{1}$, Mingyu Han ${ }^{1,2}$, Guojian Wang ${ }^{1,2}$, Yongyi Yuan ${ }^{1}$, Yu Su', Huijun Yuan', Suoqiang Zhai ${ }^{1 *}$ and Pu Dai ${ }^{1,2^{*}}$

\begin{abstract}
Background: Mitochondrial cytopathies are characterized by a large variability of clinical phenotypes and severity. The amount of mutant mitochondrial DNA (mtDNA) in a cell, called the heteroplasmy level, is an important determinant of the degree of mitochondrial dysfunction and therefore disease severity. Understanding the distribution of heteroplasmy levels across a group of offspring is an important step in understanding the inheritance of diseases. Recently, the mtDNA A1555G mutation was found to be associated with non-syndromic and drug-induced hearing loss.
\end{abstract}

Results: Here, we report five pedigrees with multiple members having the A1555G mutation and showing diverse clinical manifestations and different heteroplasmy levels. Clinical evaluations revealed that the hearing impairment phenotypes varied with respect to the severity of hearing loss, age of onset of hearing loss, and pattern of audiometric configuration. These five Chinese pedigrees had different penetrance of hearing loss, ranging from 10-52\%. A molecular study showed that the average heteroplasmy rates of the five pedigrees were $31.98 \%$ (0-91.35\%), 78.28\% (32.8-96.08\%), 87.99\% (82.32-94.65\%), 93.34\% (91.02-95.05\%), and 93.57\% (91.38-94.24\%). There was no gradual tendency of heteroplasmy to increase or decrease along with transmission. A study of the relationship between clinical features and genetic background found that the percentage of deafness was 0 when the heteroplasmy level was less than 50\%, 25\% when the heteroplasmy level was 50-80\%, 47.06\% when the heteroplasmy level was $80-90 \%$, and $57.58 \%$ when the heteroplasmy level exceeded $90 \%$. The risk of deafness rose with the heteroplasmy level.

Conclusions: The results suggest that there are large random shifts in the heteroplasmy level between mothers and offspring with the A1555G mutation; heteroplasmy could disappear randomly when the heteroplasmy level of the pedigree was low enough, and no regular pattern was found. The heteroplasmy level may be one of the factors influencing the penetrance of deafness caused by the mtDNA A1555G mutation.

Keywords: Hearing loss, Mitochondrial DNA, Heteroplasmy, A1555G mutation

\footnotetext{
*Correspondence: zhaisq@plagh.com.cn; daipu301@vip.sina.com

'Department of Otorhinolaryngology, Head and Neck Surgery, PLA General Hospital, 28\# Fuxing Road, Beijing 100853, P. R. China

${ }^{2}$ Department of Otolaryngology, Hainan Branch of PLA General Hospital, Haitang Bay, Sanya 572000, P. R. China
} 


\section{Background}

The mitochondrial $12 \mathrm{~S}$ ribosomal RNA (rRNA) gene is a hotspot for mutations associated with aminoglycosideinduced, non-syndromic hearing loss. Of these mutations, the A1555G mutation at a highly conserved decoding region of the 12S rRNA has been reported to be associated with hearing loss in many ethnic populations [1-4]. In these pedigrees and individuals, the variable clinical phenotype and incomplete penetrance of the A1555G-induced hearing loss complicates our understanding of this mutation. Many factors could be involved in the variable phenotype of the A1555G mutation; these include ethnic background, environmental influences, aminoglycoside used, nuclear genes, mitochondrial haplotypes/variants, and a possible threshold effect [5-9]. For example, the mitochondrial tRNA variants tRNA ${ }^{\text {Glu }}$ A14693G, tRNA ${ }^{\text {Arg }} \mathrm{T} 10454 \mathrm{C}$, tRNA ${ }^{\text {Thr }}$ T15908C, and tRNA ${ }^{\text {Ser(UCN) }}$ G7444A may contribute to a higher penetrance of hearing loss in pedigrees carrying the A1555G mutation $[10,11]$. In addition, as one of the most important hereditary features, the heteroplasmy level of a mitochondrial DNA (mtDNA) mutation may be another influential factor [12].

The mtDNA is inherited maternally. Heteroplasmy describes a condition in which cells containing mtDNA with pathogenic mutations also contain the normal wildtype mtDNA. The relative amount of mutant mtDNA compared with the wild-type mtDNA in a cell, called the heteroplasmy level, is an important determinant of the degree of mitochondrial dysfunction and therefore the disease severity. The risk for transmission of a mitochondrial disorder is difficult to estimate because of heteroplasmy. Therefore, elucidating the distribution of heteroplasmy levels across a group of offspring is an important step in understanding the inheritance of diseases caused by mtDNA mutations [13,14].

Here, we analyzed the clinical and genetic features of five heteroplasmy pedigrees with aminoglycoside-induced, non-syndromic hearing loss and the A1555G mutation, identified through a nationwide epidemiological survey, to explore the influence of heteroplasmy on the phenotype and inheritance of the A1555G mutation.

\section{Methods}

\section{Subjects and audiological examinations}

As part of a genetic screening program for hearing impairment, five Han Chinese families with the $12 \mathrm{~S}$ rRNA A1555G mutation were identified by the Department of Otolaryngology of the Chinese PLA General Hospital, using mitochondrial 12S rRNA sequence analysis (Figure 1). A comprehensive history and physical examination were used to identify any syndromic finding, a history of aminoglycoside use, and genetic factors related to the hearing impairment in the pedigree members. Age-appropriate audiological examinations, including pure-tone audiometry (PTA) or auditory brainstem response, immittance testing, and distortion product

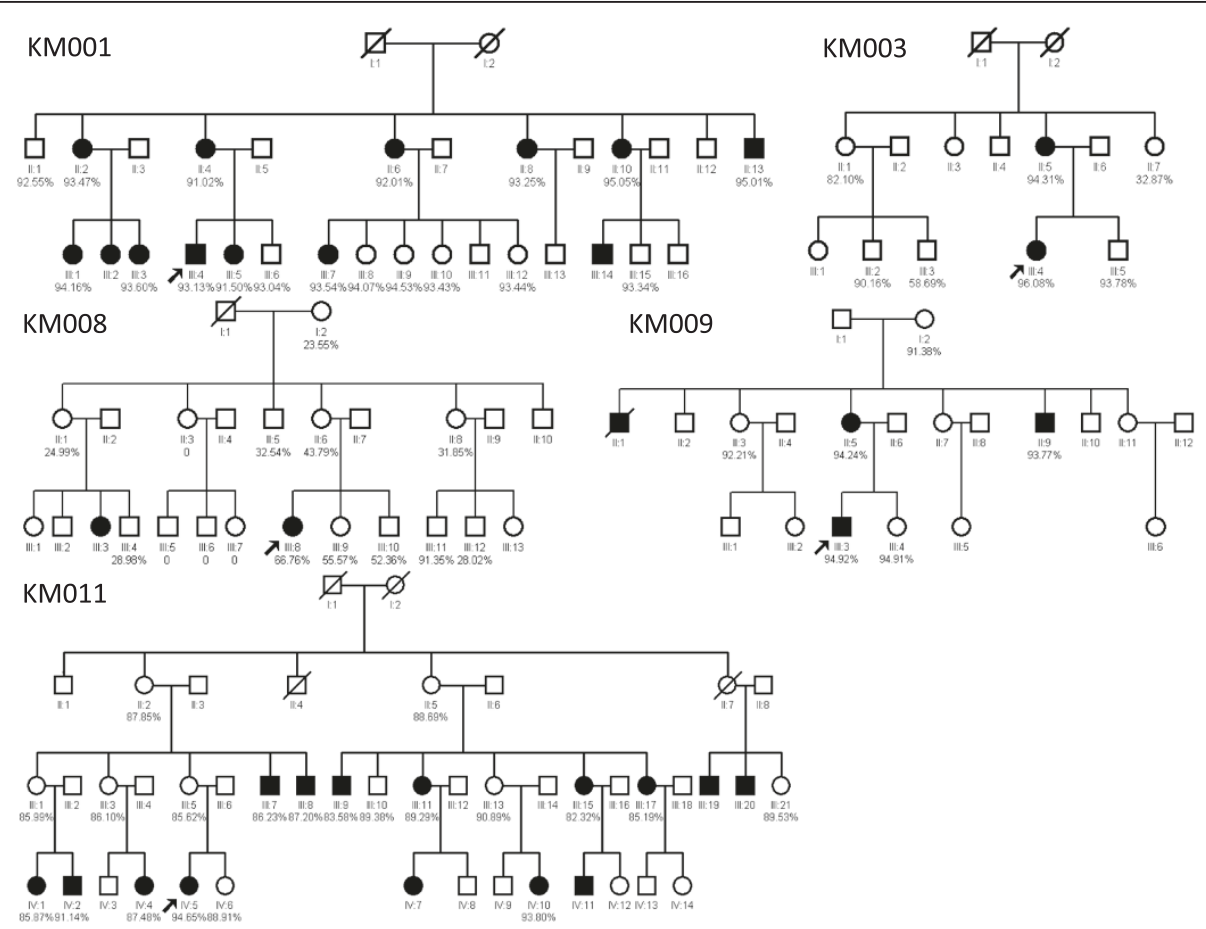

Figure 1 Five Chinese pedigrees with aminoglycoside-induced and non-syndromic hearing impairment. Hearing-impaired individuals are indicated by filled symbols. Arrow denotes probands. The heteroplasmy level is marked below the identifiers. 
otoacoustic emissions, were performed. The PTA was calculated from the sum of the audiometric thresholds at $500,1000,2000$, and $4000 \mathrm{~Hz}$. The severity of hearing impairment was classified into five grades: normal, $<26$ decibels (dB); mild, 26-40 dB; moderate, 41-70 dB; severe, 71-90 dB; and profound, >90 dB (Van Camp G. Hereditary Hearing Loss Homepage). Informed consent was obtained from the participants before their participation in the study, in accordance with the Ethics Committee of the Chinese PLA General Hospital. The pedigree maps were constructed using Cyrillic software ver. 2.1.3.1. The penetrance was calculated by dividing the affected number of matrilineal relatives by the total number of matrilineal relatives.

\section{Molecular study}

\section{Direct sequencing}

Genomic DNA was isolated from the whole blood of participants. The subjects' DNA spanning the entire mitochondrial $12 \mathrm{~S}$ rRNA gene was amplified by PCR [15]. All genotypes were detected by direct sequencing and verified by forward and reverse sequencing using an ABI 3730 automated DNA sequencer (Applied Biosystems, Foster City, CA, USA). The resultant sequence data were compared with the updated consensus Cambridge sequence (GenBank accession number: NC_012920). The mtDNA A1555G mutation was identified in peripheral blood leukocytes in every patient.

\section{SNaPshot technique}

The level of heteroplasmy in peripheral blood leukocytes was determined using SNaPshot technology (Applied Biosystems) to estimate the areas of the peaks of the wild-type (A allele peak) and mutant ( $G$ allele peak) alleles at the 1555 site. The heteroplasmy rate was calculated as the area of the G allele/(area of the A allele + area of the $\mathrm{G}$ allele). This technique involves PCR amplification of the region of interest, purification of the product, and annealing of a SNaPshot primer that ends one nucleotide $5^{\prime}$ from a known single nucleotide polymorphism (SNP). A single base extension reaction is then performed in the presence of the four fluorescently labeled dideoxynucleotide triphosphates (ddNTPs). Upon excitation with a laser, each different fluorescent dye emits a color specific for a ddNTP, i.e., green for A, blue for G, black for $\mathrm{C}$, and red for $\mathrm{T}$.

A pair of PCR primers (forward, $5^{\prime}$-CCACCTCTTGC

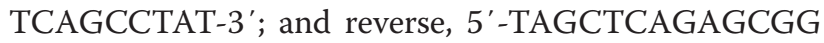
TCAAGTT-3') and a SNaPshot probe (5'-TTTTTTTT TTTTTTTTTTTTTTTTTTTCCCTACGCATTTATATA GAGGAG-3') were designed. The resulting PCR fragment was $436 \mathrm{bp}$ in size. The PCR reactions (Applied Biosystems) were performed in $40-\mu \mathrm{l}$ volumes according to the following conditions: initial denaturation at $95^{\circ} \mathrm{C}$ for
$2 \mathrm{~min}$, five cycles of $94^{\circ} \mathrm{C}$ for $20 \mathrm{~s}, 55^{\circ} \mathrm{C}$ for $30 \mathrm{~s}$, and $72^{\circ} \mathrm{C}$ for $40 \mathrm{~s}$, and then five cycles of $94^{\circ} \mathrm{C}$ for $20 \mathrm{~s}, 53^{\circ} \mathrm{C}$ for $30 \mathrm{~s}$, and $72^{\circ} \mathrm{C}$ for $40 \mathrm{~s}$, followed by 25 cycles of $94^{\circ} \mathrm{C}$ for $30 \mathrm{~s}, 52^{\circ} \mathrm{C}$ for $30 \mathrm{~s}$, and $72^{\circ} \mathrm{C}$ for $40 \mathrm{~s}$, with a final 5 -min extension at $72^{\circ} \mathrm{C}$. After the PCR, SNaPshot extension reactions were carried out on an ABI PRISM 3730 DNA sequencer (Applied Biosystems). All samples were genotyped using GeneMapper software.

\section{Mutational analysis of GJB2 gene}

The DNA fragments spanning the entire coding region of GJB2 gene were amplified by PCR using the following oligodeoxy-nucleotides: forward-5'TATGACACTCCCC AGCACAG' and reverse-5'GGGCAATGCTTAAACTG GC3'. PCR amplification and subsequent sequencing analysis were performed as detailed elsewhere. The results were compared with the wild type GJB2 sequence (GenBank accession number: M86849) to identify the mutations.

\section{Results}

\section{Clinical characteristics of the hearing-loss patients in five pedigrees}

A comprehensive history and physical and audiological examinations were performed to identify syndromic phenotypes. The history of aminoglycoside use and genetic factors related to the hearing impairment were identified in all available members of five Chinese pedigrees carrying the A1555G mutation. Comprehensive family and medical histories of probands and other members of these Chinese families showed no other clinical abnormalities, including visual dysfunction, diabetes, muscular diseases, and neurological disorders. All pedigrees had multiple affected individuals (Figure 1). There were 36 individuals with aminoglycoside-induced, non-syndromic bilateral hearing loss in these five pedigrees, including seven individuals from whom no blood samples were obtained (KM001-III-2, KM001-III-14, KM008-III-3, KM011-III-19, KM011-III-20, KM011-IV-7, and KM011IV-11), one who had died (KM009-II-1), and two who were lacking hearing test results (KM003-II-5 and KM009-II-9). The remaining 26 individuals with aminoglycosideinduced or non-syndromic bilateral hearing loss had different clinical characteristics (Table 1). The age of these 26 individuals ranged from 18 to 52 years. The age of onset of the hearing loss ranged from less than one year to 20 years in 13 patients, while the other 13 individuals or their family members did not remember the specific time of onset. All had a history of receiving aminoglycoside antibiotics, except five individuals who were not certain (Table 1). Meanwhile all normal hearing members carrying the $1555 \mathrm{~A}>\mathrm{G}$ mutation have no history of aminoglycosides usage. Audiological evaluations revealed symmetric hearing impairment in all 26 
Table 1 Summary of clinical data on deafness in five Chinese pedigrees carrying a heteroplasmic A1555G mutation

\begin{tabular}{|c|c|c|c|c|c|c|c|c|c|}
\hline Subject & Gender & Age & $\begin{array}{l}\text { Audiometric } \\
\text { configuration }\end{array}$ & $\begin{array}{c}\text { Use of } \\
\text { aminoglycosides }\end{array}$ & $\begin{array}{l}\text { Age at } \\
\text { onset }(y)\end{array}$ & $\begin{array}{l}\text { PTA (dB) } \\
\text { right ear }\end{array}$ & $\begin{array}{l}\text { PTA (dB) } \\
\text { left ear }\end{array}$ & $\begin{array}{c}\text { Level of } \\
\text { hearing } \\
\text { impairment }\end{array}$ & $\begin{array}{l}\text { Heteroplasmy } \\
\text { rate }(\%)\end{array}$ \\
\hline KM001-II-2 & $\mathrm{F}$ & 50 & Slope & Yes & 1 & 76 & 70 & Severe & 93.47 \\
\hline KM001-II-4 & $\mathrm{F}$ & 45 & Slope & Yes & Not known & 40 & 51 & Mild & 91.02 \\
\hline KM001-II-6 & $\mathrm{F}$ & 43 & Slope & Yes & Not known & 41 & 41 & Moderate & 92.01 \\
\hline KM001-II-8 & $\mathrm{F}$ & 41 & Slope & Not known & Not known & 76 & 63 & Moderate & 93.25 \\
\hline KM001-II-10 & $\mathrm{F}$ & 39 & Slope & Not known & Not known & 40 & 39 & Mild & 95.05 \\
\hline KM001-II-13 & M & 36 & Slope & Yes & Not known & 54 & 60 & Moderate & 95.01 \\
\hline KM001-III-1 & $\mathrm{F}$ & 29 & Slope & Yes & 1.5 & 74 & 74 & Severe & 94.16 \\
\hline KM001-III-3 & $\mathrm{F}$ & 23 & Slope & Yes & 2 & 93 & 95 & Profound & 93.60 \\
\hline KM001-III-4 & M & 24 & Slope & Yes & 1 & 95 & 93 & Profound & 93.13 \\
\hline KM001-III-5 & $F$ & 22 & Slope & Yes & Not known & 43 & 54 & Moderate & 91.50 \\
\hline KM001-III-7 & $\mathrm{F}$ & 22 & Slope & Yes & 12 & 50 & 70 & Moderate & 93.54 \\
\hline KM003-III-4 & $F$ & 18 & Slope & Yes & Not known & 103 & 100 & Profound & 96.08 \\
\hline KM008-III-8 & $\mathrm{F}$ & 19 & Slope & Yes & 2 & 104 & 109 & Profound & 66.76 \\
\hline KM009-II-5 & $\mathrm{F}$ & 44 & Slope & Yes & Not known & 98 & 76 & Severe & 94.24 \\
\hline KM009-III-3 & M & 20 & Slope & Yes & Not known & 98 & 95 & Profound & 94.92 \\
\hline KM011-III-7 & M & 35 & Slope & Yes & 6 & 83 & 80 & Severe & 86.23 \\
\hline KM011-III-8 & M & 30 & Flat & Not known & 20 & 59 & 68 & Moderate & 87.20 \\
\hline KM011-1II-9 & M & 52 & Slope & Yes & 3 & 100 & 100 & Profound & 83.58 \\
\hline KM011-III-11 & $F$ & 45 & Slope & Yes & Not known & 51 & 55 & Moderate & 89.29 \\
\hline KM011-III-15 & $\mathrm{F}$ & 40 & Flat & Yes & 3 & 110 & 110 & Profound & 82.32 \\
\hline KM011-III-17 & $\mathrm{F}$ & 37 & Slope & Yes & 4 & 69 & 73 & Moderate & 85.19 \\
\hline KM011-IV-1 & $\mathrm{F}$ & 26 & Slope & Not known & Not known & 46 & 49 & Moderate & 85.87 \\
\hline KM011-IV-2 & M & 24 & Slope & Not known & Not known & 51 & 48 & Moderate & 91.14 \\
\hline KM011-IV-4 & $\mathrm{F}$ & 20 & Slope & Yes & 2 & 109 & 103 & Profound & 87.48 \\
\hline KM011-IV-5 & $\mathrm{F}$ & 20 & Slope & Yes & Not known & 93 & 93 & Profound & 94.65 \\
\hline KM011-IV-10 & $\mathrm{F}$ & 20 & Flat & Yes & 2 & 94 & 93 & Profound & 93.80 \\
\hline
\end{tabular}

individuals (Figure 2). However, the subjects exhibited variable severity of the hearing impairment, with two, ten, four, and ten subjects having mild, moderate, severe, and profound hearing impairment, respectively. The subjects also had different audiometric configurations; two subjects had flat patterns, and 24 had slope patterns. The five Chinese pedigrees exhibited different hearing loss penetrance: $52 \%, 18.2 \%, 10 \%, 26.7 \%$, and $44.1 \%$ (Table 2), with an average penetrance of $30.2 \%$.

\section{Mutational screening of the 12S rRNA gene in maternal subjects}

To elucidate the molecular basis of the hearing loss, we initially performed a mutational analysis of the mitochondrial $12 \mathrm{~S}$ rRNA gene in all affected maternally related individuals in the five pedigrees who were seen in the Department of Otolaryngology of the Chinese PLA General Hospital. Initially, 66 individuals were detected. DNA spanning the $12 \mathrm{~S}$ rRNA from each subject was
PCR amplified and sequenced. Sixty-two individuals harbored a heteroplasmic mtDNA A1555G mutation in the $12 \mathrm{~S}$ rRNA gene, with different relative sizes of the A and G peaks (Figure 3). The other four individuals appeared to have no A1555G mutation (KM008-II-3, KM008-III5, KM008-III-6, and KM008-III-7), as we did not find any $\mathrm{G}$ mutation peak in the direct sequencing graphs of these four individuals. However, if the heteroplasmy rate was too high or too low, we would not have been able to distinguish homoplasmy, heteroplasmy, or wild type using standard Sanger sequencing technology.

\section{Mutational analysis of GJB2}

To examine the role of GJB2 gene in the phenotypic expression of the A1555G mutation, we performed the mutational screening of GJB2 gene in probands of these five Chinese pedigrees. No GJB2 mutation was found. Indeed, the absence of a GJB2 gene mutation in these hearing impaired subjects suggested that the GJB2 gene 

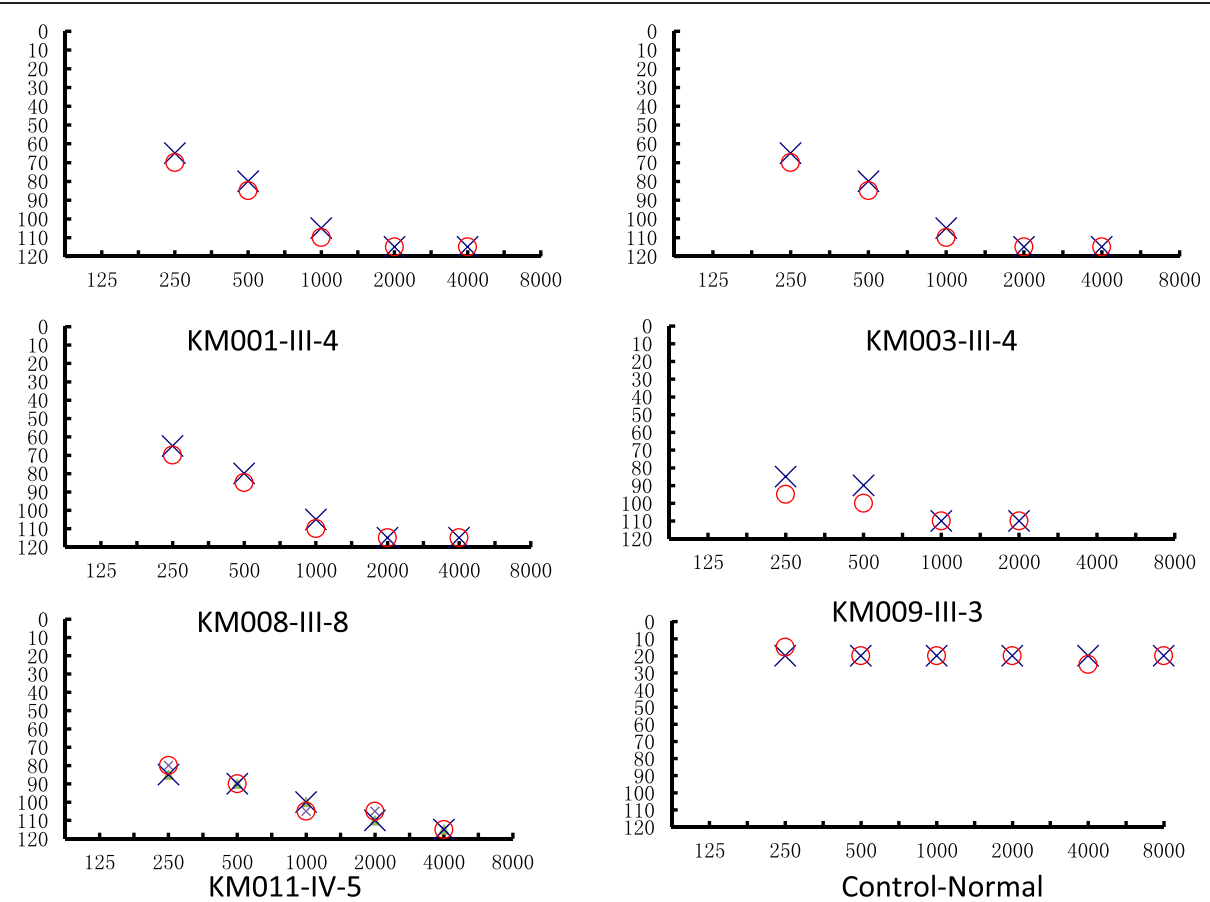

Figure 2 Air conduction audiogram of 5 affected probands with the A1555G mutation and one Chinese control. Symbols: X-left ear, O-right ear.

might not modify the phenotypic effects of the A1555G mutation in them.

Analysis of the heteroplasmy levels of family members To identify the A1555G mutation and explore the heteroplasmy inheritance pattern, we used the SNaPshot technique to quantify the heteroplasmy level. The average heteroplasmy rates differed among the five pedigrees (Table 2, Figures 1 and 3). The average heteroplasmy rate of the KM001 pedigree was 93.34\% (range, 91.02-95.05\%); the average heteroplasmy rates of generations II and III in the KM001 pedigree were $91.19 \%$ and $91.43 \%$, respectively. The average heteroplasmy rate of the KM003 pedigree was $78.28 \%$ (range, 32.87-96.08\%), with average

Table 2 Summary of genetic and molecular data for 5 Chinese families carrying the heteroplasmy A1555G mutation

\begin{tabular}{|c|c|c|c|c|c|c|c|c|c|}
\hline \multirow[t]{2}{*}{ Pedigree } & \multirow{2}{*}{$\begin{array}{c}\text { Number of } \\
\text { matrilineal } \\
\text { relatives }\end{array}$} & \multirow{2}{*}{$\begin{array}{c}\text { Number } \\
\text { of patients }\end{array}$} & \multirow{2}{*}{$\begin{array}{c}\text { Penetrance }^{a} \\
(\%)\end{array}$} & \multirow[t]{2}{*}{ Generation } & \multirow{2}{*}{$\begin{array}{l}\text { Number of } \\
\text { generation }\end{array}$} & \multicolumn{4}{|c|}{ Heteroplasmy level $^{\mathbf{b}}(\%)$} \\
\hline & & & & & & Min & Max & Generation mean & Pedigree mean \\
\hline \multirow[t]{2}{*}{ KM001 } & 25 & 13 & 52 & $\|$ & 7 & 91.02 & 95.05 & 93.19 & 93.34 \\
\hline & & & & III & 11 & 91.50 & 94.16 & 93.43 & \\
\hline \multirow[t]{2}{*}{ KM003 } & 11 & 2 & 18.2 & $\|$ & 3 & 32.87 & 94.31 & 69.76 & 78.28 \\
\hline & & & & III & 4 & 58.69 & 96.08 & 84.68 & \\
\hline \multirow[t]{3}{*}{ KM008 } & 20 & 2 & 10 & । & 1 & 23.55 & 23.55 & 23.55 & 31.98 \\
\hline & & & & $\|$ & 5 & 0 & 43.79 & 26.63 & \\
\hline & & & & III & 9 & 0 & 91.35 & 35.89 & \\
\hline \multirow[t]{3}{*}{ KM009 } & 15 & 4 & 26.7 & । & 1 & 91.38 & 91.38 & 91.38 & 93.57 \\
\hline & & & & $\|$ & 3 & 92.21 & 94.24 & 93.41 & \\
\hline & & & & III & 2 & 94.41 & 94.92 & 94.67 & \\
\hline \multirow[t]{3}{*}{ KM011 } & 34 & 15 & 44.1 & $\|$ & 2 & 87.85 & 88.69 & 88.27 & 87.99 \\
\hline & & & & III & 12 & 82.32 & 90.89 & 86.78 & \\
\hline & & & & IV & 6 & 85.87 & 94.65 & 90.31 & \\
\hline
\end{tabular}




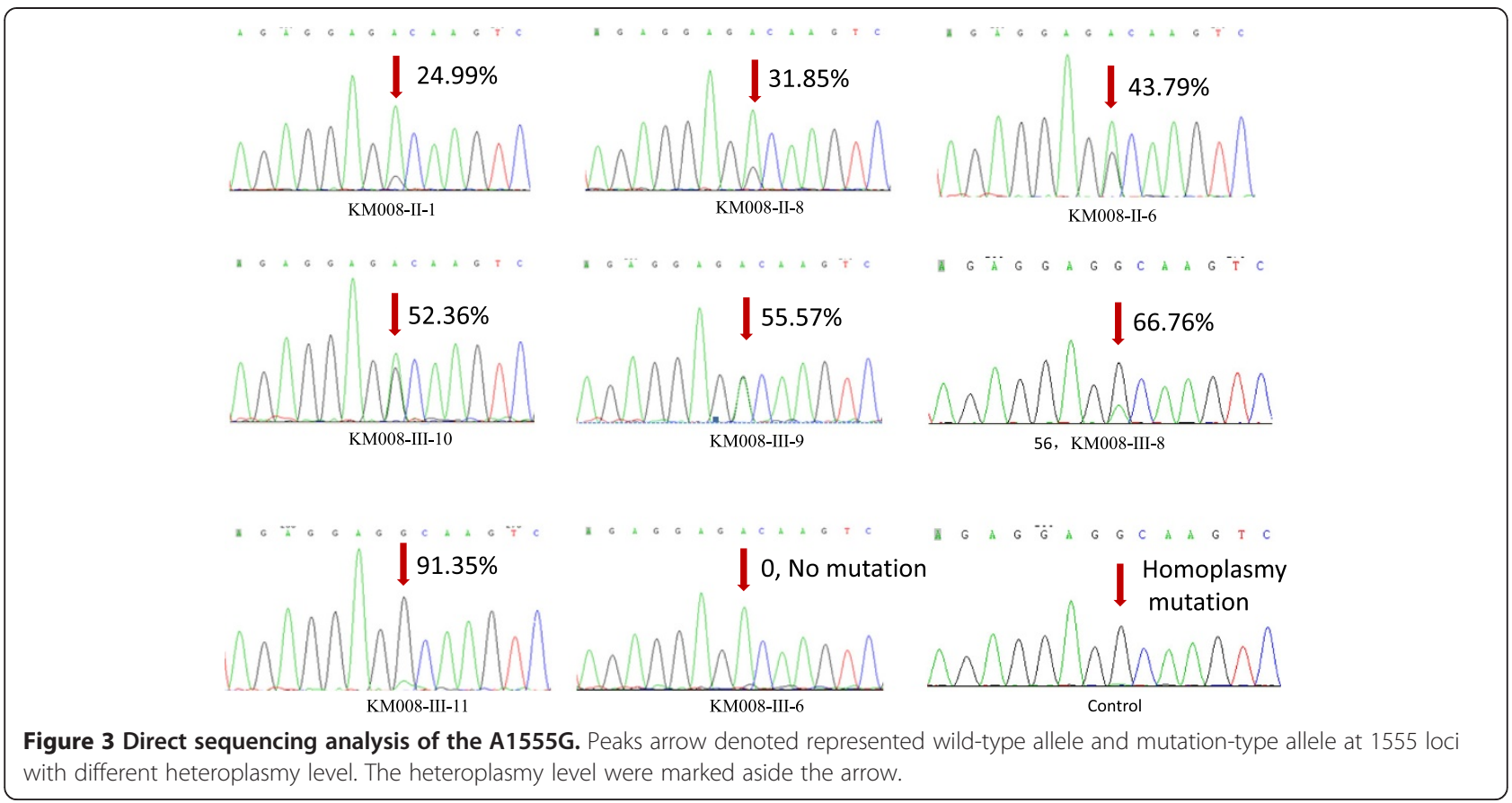

heteroplasmy rates of $69.76 \%$ and $84.68 \%$ in generations II and III, respectively. For the KM008 pedigree, the average heteroplasmy rate was $31.98 \%$ (range, $0-91.35 \%$ ), and the average heteroplasmy rates of generations I to III were $23.55 \%, 26.63 \%$, and $35.89 \%$, respectively. The KM009 pedigree had an average heteroplasmy rate of $93.57 \%$ (range, 91.38-94.24\%); the average heteroplasmy rates of generations I to III were $91.38 \%, 93.41 \%$, and $94.67 \%$, respectively. The average heteroplasmy rate of the KM011 pedigree was $87.99 \%$ (range, $82.32-94.65 \%$ ), and the average heteroplasmy rates of generations II to IV were $88.27 \%, 86.78 \%$, and $90.31 \%$, respectively.

The KM008-III pedigree included four members (KM008-II-3, KM008-III-5, KM008-III-6, and KM008III-7) with no A1555G mutation based on direct sequencing. The mother of KM008-II-3 was KM008-I-2, whose mtDNA A1555G heteroplasmy rate was $23.55 \%$. The heteroplasmy rates of the brothers and sisters of KM008-II-3 ranged from 24.99-3.79\%. The offspring of KM008-II-3 (KM008-III-5, KM008-III-6, and KM008-III-7) had no mtDNA A1555G mutation, like their mother (KM008-II-3), and they all had normal hearing. We did not find any relationship that could explain the increases or decreases in the mtDNA A1555G heteroplasmy levels of the different generations of these five pedigrees.

\section{Clinical and genetic evaluation of the five pedigrees with the A1555G mutation}

To explore the relationship between the clinical features and heteroplasmy levels in the pedigrees, the hearing loss levels of five probands in the five pedigrees, including
KM001-III-4, KM003-III-4, KM008-III-8, KM009-III-3, and KM011-IV-5, and their heteroplasmy levels were analyzed. All five probands had symmetric, profound, slope pattern hearing impairment (Table 1 and Figure 2). Their respective heteroplasmy levels were 93.13, 96.08, 66.76, 94.92, and $94.65 \%$, and the average heteroplasmy levels of the pedigrees were $93.34,78.28,31.98,93.57$, and $87.99 \%$, respectively (Table 2).

Among individuals, two had mild hearing loss, the average age of them was 42 years, their heteroplasmy levels were $91.02 \%$ and $95.05 \%$, with an average of 93.04\%. Ten had moderate hearing loss, the average age of these members was 32.6 years, their heteroplasmy levels ranged from $85.19 \%$ to $95.01 \%$ and averaged $90.36 \%$. Four had severe hearing loss, the average age was 39.5 years, with heteroplasmy levels of 93.47, 94.16, 94.24 , and $86.23 \%$, and an average of $92.05 \%$. Another 10 individuals had profound hearing loss, the average age of these 10 members was 25.6 years, their heteroplasmy levels ranged from $66.76 \%$ to $96.08 \%$ and averaged $88.63 \%$. No regular pattern was identified (Table 1).

We also analyzed the percentage of deafness in all maternally related members according to the heteroplasmy level (Figure 1). In our pedigrees, four members lacked the mtDNA A1555G mutation (KM008-II-3, KM008-III-5, KM008-III-6, and KM008-III-7); all had normal hearing. Eight members had heteroplasmy levels of $<50 \%$ (KM003II-7, KM008-I-2, KM008-II-1, KM008-II-5, KM008-II-6, KM008-II-8, KM008-III-4, and KM008-III-12); they all also had normal hearing. Another four had heteroplasmy levels from 50\% to 80\% (KM003-III-3, KM008- 
III-8 KM008-III-9, and KM008-III-10). Only one (KM008-III-8, with a heteroplasmy level of 66.76\%) was deaf, giving a $25 \%$ risk for deafness associated with this heteroplasmy level. Seventeen members in the five pedigrees had heteroplasmy levels of $80-90 \%$, eight of whom had deafness for a risk of $47.06 \%$. The heteroplasmy level of the remaining 33 members exceeded $90 \%$, and 19 had deafness, for a risk of $57.58 \%$. Thus, the risk for deafness tended to increase with an increase in the heteroplasmy level.

\section{Discussion}

This study examined the clinical, genetic, and molecular characteristics of five Chinese pedigrees with aminoglycosideinduced, non-syndromic hearing impairment associated with the heteroplasmic A1555G mutation and analyzed the levels and inheritance of heteroplasmy for this mutation.

Hearing impairment as the sole clinical phenotype was present only in the maternal lineages of these pedigrees with the A1555G mutation. The hearing impairment phenotype and penetrance varied among the pedigrees, as reported for other pedigrees [5-9]. In our subjects, the onset age of hearing impairment ranged from younger than 1 year old to 20 years old. The onset of hearing loss was related to the use of aminoglycosides.

The direct sequencing mutation analysis initially revealed the heteroplasmy trait of the A1555G mutation in probands and their pedigrees (Figure 3). However, not all individuals showed heteroplasmy by direct sequencing because the mixed peak influenced the results, especially when the heteroplasmy was very low or high. To accurately determine the heteroplasmy level, we used the SNaPshot technique to quantify the heteroplasmy level. This technique is used specifically to genotype single nucleotide polymorphisms (SNPs). According to previous studies, the SNaPshot technique is an accurate, sensitive, flexible, and cost-effective method for examining many mutations or SNPs in one run of the SNaPshot assay $[16,17]$. It is also an accurate, reproducible, and sensitive method for determining heteroplasmic mtDNA mutations in different tissues, and it is a promising system for use in the pre- and postnatal diagnosis of mtDNA-associated disorders [18]. In the present study, the heteroplasmy level could be calculated reliably using the SNaPshot technique, which provides a technical platform for the quantitative detection of the mtDNA A1555G mutation.

We identified variable transmission of heteroplasmy across different generations in the five Chinese pedigrees. Within each pedigree, the heteroplasmy level differed among individuals and among generations. This may be explained by the peculiarities of mtDNA, i.e., different mutation loads can occur in different family members with mitotic segregation in a cell [19]. Although the average heteroplasmy tended to increase in generations, except generation III of KM011, there was no observed tendency for an increase or decrease across individuals, not only from mothers to children but also in siblings. For example, the heteroplasmy level of KM003-II-1 was $82.10 \%$, that of her son KM003-III-2 was $90.16 \%$, and that of the other son was $58.69 \%$ (Figure 1). In the KM008 pedigree, four members (KM008-II-3, KM008III-5, KM008-III-6, and KM008-III-7) had no mutation at all. The mother of KM008-II-3 was KM008-I-2, whose heteroplasmy rate was 23.55\%. KM008-II-3 did not inherit any A1555G mutation, and all her offspring lacked the mtDNA A1555G mutation (KM008-III-5, KM008-III-6, and KM008-III-7) and had normal hearing. This phenomenon may be attributable to the socalled "mitochondrial bottleneck" and "random allocation" $[20,21]$, meaning that a small number of mutated mtDNA molecules become the founders for the offspring's mtDNA when mitosis takes place. When the number of segregating units (groups of clonal mtDNA that co-segregate) that become the mtDNA founders of the embryo is small, large fluctuations in heteroplasmy may occur in a single generation.

To explore the relationship between clinical features and genetic backgrounds, we analyzed the auditory threshold of hearing loss and heteroplasmy level of the mtDNA A1555G mutation within pedigrees and among members, as well as the percentage of all maternally related members with deafness according to different heteroplasmy levels. The analysis indicated that the risk for deafness increased with an increase in the heteroplasmy level. The risk for deafness was 0 for members with heteroplasmy levels $<50 \%, 25 \%$ for members with heteroplasmy levels from $50-80 \%, 47.06 \%$ for members with heteroplasmy levels from $80-90 \%$, and $57.58 \%$ for members with heteroplasmy levels exceeding 90\%. In this heredity mitochondrial trait, there was a threshold effect regarding the onset of disease. In our study, all subjects with heteroplasmy levels $<50 \%$ had normal hearing, and $25 \%$ of the subjects with heteroplasmy levels between $50-80 \%$ had hearing loss. This implies that the heteroplasmy level of the mtDNA A1555G mutation must exceed $50 \%$ to cause the hearing-loss phenotype. Further functional studies should confirm this. Given that the risk for deafness increased with the heteroplasmy level, the heteroplasmy level may influence the penetrance of deafness related to the mtDNA A1555G mutation, as seen with other factors, including ethnic background, environment, aminoglycoside use, nuclear genes, mitochondrial haplotypes/variants, and the threshold effect [5-9].

Based on our data, the homoplasmy variation, including that for the A1555G mutation, is transmitted stably in all maternal lineages of a pedigree based on mitochondrial maternal hereditary; however, the heteroplasmy variation 
is likely to involve more complicated genetic and clinical factors. The risk for deafness increased with the heteroplasmy level, which also appeared to influence the penetrance of deafness associated with the mtDNA A1555G mutation.

\section{Conclusions}

There were large, seemingly random differences in the heteroplasmy level between mothers and offspring in the process of maternal transmission. The heteroplasmy could disappear when the heteroplasmy level of pedigree was sufficiently low. No regular pattern was identified, other than the tendency for an increased risk for deafness with an increase in the heteroplasmy level. The heteroplasmy level may also influence the penetrance of deafness related to the mtDNA A1555G mutation.

\section{Competing interests}

The authors declare that they have no competing interests.

\section{Authors' contributions}

Conceived and designed the experiments: PD YHZ SQZ. Performed the experiments: YHZ DYK MYH GJW YYY YS. Analyzed the data: YHZ SSH HJY SQZ PD. Contributed reagents/materials/analysis tools: YHZ SSH. Wrote the paper: YHZ SSH PD. All authors read and approve the final manuscript.

\section{Authors' information}

Yuhua Zhu and Shasha Huang as co-first authors.

\section{Acknowledgments}

This work was supported by the Chinese National Nature Science Foundation Research Grant $(81000414,81370021)$ and Beijing Nova Programme(Z131107000413102) to Dr. Yuhua Zhu. Chinese National Nature Science Foundation Research Grant (31071099, 81230020), grant from State 863 High Technology R\&D Key Project of China (2011AA02A112), grant from Minister of Health of China (201202005) and grant from the Minister of Science and Technology (2012BAl09B02) to Dr. Pu Dai.

Received: 3 November 2013 Accepted: 12 February 2014 Published: 17 February 2014

\section{References}

1. Dzhemileva LU, Posukh OL, Tazetdinov AM, Barashkov NA, Zhuravskiĭ SG, Ponidelko SN, Markova TG, Tadinova VN, Fedorova SA, Maksimova NR, Khusnutdinova EK: Analysis of mitochondrial 12S rRNA and tRNA(Ser (UCN)) genes in patients with nonsyndromic sensorineural hearing loss from various regions of Russia. Genetika 2009, 45(7):982-991.

2. Human H, Lombard D, de Jong G, Bardien S: A South African family with the mitochondrial A1555G mutation on haplogroup LOd. Biochem Biophys Res Commun 2009, 382(2):390-394.

3. Bai Y, Wang Z, Dai W, Li Q, Chen G, Cong N, Guan M, Li H: A six-generation Chinese family in haplogroup B4C1C exhibits high penetrance of 1555A $>$ G-induced hearing Loss. BMC Med Genet 2010, 11:129.

4. Bae JW, Kim DB, Choi JY, Park HJ, Lee JD, Hur DG, Bae SH, da Jung J, Lee $\mathrm{SH}$, Kim UK, Lee KY: Molecular and clinical characterization of the variable phenotype in Korean families with hearing loss associated with the mitochondrial A1555G mutation. PLoS One 2012, 7(8):42463.

5. de Moraes VC, Alexandrino F, Andrade PB, Câmara MF, Sartorato EL: Study of modifiers factors associated to mitochondrial mutations in individuals with hearing impairment. Biochem Biophys Res Commun 2009, 381(2):210-213.

6. Ding $Y, L i Y$, You J, Yang L, Chen B, Lu J, Guan MX: Mitochondrial tRNA (Glu) A14693G variant may modulate the phenotypic manifestation of deafness-associated $12 S$ rRNA A1555G mutation in a Han Chinese family. J Genet Genomics 2009, 36(4):241-250.
7. Qian Y, Guan MX: Interaction of aminoglycosides with human mitochondrial $12 \mathrm{~S}$ rRNA carrying the deafness-associated mutation. Antimicrob Agents Chemother 2009, 53(11):4612-4618.

8. Lu J, Qian Y, Li Z, Yang A, Zhu Y, Li R, Yang L, Tang X, Chen B, Ding Y, Li Y, You J, Zheng J, Tao Z, Zhao F, Wang J, Sun D, Zhao J, Meng Y, Guan MX: Mitochondrial haplotypes may modulate the phenotypic manifestation of the deafness-associated $12 \mathrm{~S}$ rRNA $1555 \mathrm{~A}>\mathrm{G}$ mutation. Mitochondrion 2010, 10(1):69-81.

9. Guan MX: Mitochondrial 125 rRNA mutations associated with aminoglycoside ototoxicity. Mitochondrion 2011, 11(2):237-245.

10. Yuan H, Qian Y, Xu Y, Cao J, Bai L, Shen W, Ji F, Zhang X, Kang D, Mo JQ, Greinwald JH, Han D, Zhai S, Young WY, Guan MX: Cosegregation of the G7444A mutation in the mitochondrial COI/RNA(Ser(UCN)) genes with the $12 S$ rRNA A1555G mutation in a Chinese family with aminoglycoside-induced and non-syndromic hearing loss. Am J Med Genet A 2005, 138A(2):133-140.

11. Young WY, Zhao L, Qian Y, Li R, Chen J, Yuan H, Dai P, Zhai S, Han D, Guan $M X$ : Variants in mitochondrial tRNAGlu, tRNAArg, and tRNAThr may influence the phenotypic manifestation of deafness associated 12S rRNA A1555G mutation in three Han Chinese families with hearing loss. Am J Med Genet A 2006, 140(20):2188-2197.

12. del Castillo FJ, Rodríguez-Ballesteros M, Martín Y, Arellano B, Gallo-Terán J, Morales-Angulo C, Ramírez-Camacho R, Cruz Tapia M, Solanellas J, Martínez-Conde A, Villamar M, Moreno-Pelayo MA, Moreno F, del Castillo I: Heteroplasmy for the $1555 \mathrm{~A}>\mathrm{G}$ mutation in the mitochondrial $12 \mathrm{~S}$ rRNA gene in six Spanish families with non-syndromic hearing loss. J Med Genet 2003, 40(8):632-636.

13. Wonnapinij P, Chinnery PF, Samuels DC: The distribution of mitochondrial DNA heteroplasmy due to random genetic drift. Am J Hum Genet 2008, 83(5):582-593.

14. Poulton J, Chiaratti MR, Meirelles FV, Kennedy S, Wells D, Holt IJ: Transmission of mitochondrial DNA diseases and ways to prevent them. PloS Genet 2010, 6(8):e1001066.

15. Rieder MJ, Taylor SL, Tobe VO, Nickerson DA: Automating the identification of DNA variations using quality-based fluorescence resequencing: analysis of the human mitochondrial genome. Nucleic Acid Res 1998, 26(4):967-973.

16. Bardien $\mathrm{S}$, Human H, Harris T, Hefke G, Veikondis R, Schaaf HS, van der Merwe L, Greinwald JH, Fagan J, de Jong G: A rapid method for detection of five known mutations associated with aminoglycoside-induced deafness. BMC Med Genet 2009, 10:2.

17. Wu CC, Lu YC, Chen PJ, Liu AY, Hwu WL, Hsu CJ: Application of SNaPshot multiplex assays for simultaneous multigene mutation screening in patients with idiopathic sensorineural hearing impairment. Laryngoscope 2009, 119(12):2411-2416

18. Cassandrini D, Calevo MG, Tessa A, Manfredi G, Fattori F, Meschini MC, Carrozzo R, Tonoli E, Pedemonte M, Minetti C, Zara F, Santorelli FM, Bruno $C$ : A new method for analysis of mitochondrial DNA point mutations and assess levels of heteroplasmy. Biochem Biophys Res Commun 2006, 342(2):387-393.

19. Finsterer J: Genetic, pathogenetic, and phenotypic implications of the mitochondrial A3243G tRNALeu(UUR) mutation. Acta Neurol Scand 2007, 116(1):1-14.

20. Poulton J: Transmission of mtDNA: cracks in the bottleneck. Am J Hum Genet 1995, 57(2):224-226.

21. Poulton J, Macaulay V, Marchington DR: Mitochondrial genetics '98 is the bottleneck cracked? Am J Hum Genet 1998, 62(4):752-757.

doi:10.1186/1471-2156-15-26

Cite this article as: Zhu et al: Analysis of the heteroplasmy level and transmitted features in hearing-loss pedigrees with mitochondrial 125 rRNA A1555G mutation. BMC Genetics 2014 15:26. 